BULLETIN Bulletin hispanique

HISPANIQUE Université Michel de Montaigne Bordeaux

117-2 | 2015

Métamorphose(s) : représentations et réécritures

\title{
Métamorphoses des Héliades dans quelques sonnets, de Garcilaso à Góngora
}

Sandra Contamina

\section{(2) OpenEdition}

Journals

Édition électronique

URL : http://journals.openedition.org/bulletinhispanique/3984

DOI : 10.4000/bulletinhispanique.3984

ISSN : 1775-3821

Éditeur

Presses universitaires de Bordeaux

Édition imprimée

Date de publication : 15 décembre 2015

Pagination : 473-484

ISBN : $979-10-300-0041-2$

ISSN : 0007-4640

Référence électronique

Sandra Contamina, "Métamorphoses des Héliades dans quelques sonnets, de Garcilaso à Góngora », Bulletin hispanique [En ligne], 117-2 | 2015, mis en ligne le 15 décembre 2018, consulté le 07 mai 2019.

URL : http://journals.openedition.org/bulletinhispanique/3984; DOI : 10.4000/bulletinhispanique.3984 


\title{
Métamorphoses des Héliades dans quelques sonnets, de Garcilaso à Góngora
}

\author{
SANDra Contamina \\ Université d'Angers
}

À propos du motif de la métamorphose des Héliades dans trois sonnets de Góngora, il existe une intime correspondance entre le matériau littéraire hérité d'Ovide et l'élaboration d'une écriture poétique marquée tant par la variation autour d'un thème unique que par un effacement de la matérialité du corps dans le temps de sa métamorphose.

Mots-clés : Góngora, Héliades, variation.

A propósito del motivo de la metamorfosis de las Helíades en tres sonetos de Góngora, existe une íntima relación entre el material literario heredado de Ovidio y la elaboración de una escritura poética marcada tanto por la variación alrededor de un tema único como por la desaparición de la materialidad del cuerpo en el tiempo de su metamorfosis.

Palabras claves: Góngora, Helíades, variación.

The motive of the metamorphosis of the Heliades in three of Gongora's sonnets shows a close relationship between the literary material inherited from Ovid and the elaboration of a poetical style, both marked by the variation around a unique theme and the effacing of the body's materiality at the time of its metamorphosis.

Keywords: Góngora, Heliades, variation.

T 'épisode de la métamorphose des Héliades, se trouve au début du livre II d'Ovide. Si ce livre débute par le célèbre récit de l'aventure de Phaéton luimême avec l'épisode de sa visite au palais de son père, cette évocation trouve 
son aboutissement thématique dans la transformation de ses sœurs en peupliers et de son ami Cygnus en oiseau. Voici le récit d'Ovide, traduit par Georges Lafaye $^{1}$ :

Après avoir exhalé toutes les plaintes que devait lui inspirer une telle catastrophe, Clymène en deuil, éperdue, déchirant son sein, parcourut tout l'univers; elle chercha d'abord le corps inanimé de son fils, puis ses ossements; elle les trouva enfin, mais ensevelis sur une terre étrangère ; elle se prosterna et, ayant lu son nom, elle arrosa le marbre de ses pleurs et le réchauffa de sa poitrine découverte. Les Héliades ne sont pas moins désolées; elles offrent à la mort de leur frère le vain tribut de leurs larmes; elles se frappent la poitrine de leurs mains et, comme si Phaéton pouvait entendre leurs plaintes lamentables, nuit et jour elles l'appellent, étendues au bord de son tombeau. Quatre fois la Lune entre ses cornes rapprochées avait rempli son disque ; et elles, suivant leur habitude (car le temps en avait fait une habitude), elles avaient poussé des cris de désespoir. L'une des sœurs, Phaétuse, la plus âgée, qui voulait se prosterner sur la terre, se plaignit que ses pieds étaient devenus rigides; en s'efforçant d'aller jusqu'à elle, la blanche Lampétie, se sentit tout à coup retenue par une racine; la troisième voulait s'arracher les cheveux et ses mains détachent des feuilles de sa tête ; l'une gémit de voir ses jambes immobilisées sous la forme d'un tronc, l'autre de voir ses bras changés en longs rameaux. Tandis qu'elles s'étonnent, l'écorce enveloppe leurs aines; par degré elle emprisonne leur ventre, leur poitrine, leurs épaules et leurs mains; seule restait encore libre leur bouche, appelant leur mère. Et que pourrait faire leur mère, sinon courir çà et là, où la mène l'emportement de sa douleur, et, pendant qu'il en est temps, unir ses baisers à ceux de ses filles? C'est trop peu encore : elle essaie d'arracher leurs corps aux troncs qui les enferment et elle brise avec ses mains les rameaux tendres; mais il en sort, comme d'une plaie, des gouttes de sang : "Arrête, je t'en conjure, ma mère, s'écrie chacune de celles qu'elle a blessées; arrête je t'en conjure ; c'est notre corps que tu déchires dans un arbre. Et maintenant, adieu ! ' L'écorce a gagné leur visage sur ces dernières paroles. De là coulent les larmes que distillent leurs jeunes rameaux, ces gouttes d'ambre, durcies au soleil, que reçoit le fleuve limpide et qu'il envoie aux jeunes femmes du Latium pour qu'elles en fassent leur parure.

Góngora a repris le motif de la métamorphose des Héliades dans deux sonnets. Mais avant d'en arriver à l'interprétation gongorine, il convient de retracer une part de l'itinéraire poétique de l'épisode de Phaéton, simplement pour faire le constat de l'absence criante de ces figures féminines.

Garcilaso déjà avait intégré l'évocation de leur métamorphose à l'un de ses sonnets, le sonnet XII «Si para refrenar este deseo » :

\author{
Si para refrenar este deseo \\ loco, imposible, vano, temeroso, \\ $y$ guarecer de un mal tan peligroso, \\ que es darme a entender yo lo que no creo, \\ no me aprovecha verme cual me veo, \\ o muy aventurado o muy medroso, \\ en tanta confusión que nunca oso \\ fiar el mal de mi que lo poseo,
}

1. Ovide, Métamorphoses, trad. de Georges Lafaye, Paris, Les Belles Lettres, coll. « Guillaume Budé », 1995, p. 48-49. 


\author{
¿qué me ha de aprovechar ver la pintura \\ de aquel que con las alas derretidas \\ cayendo, fama y nombre al mar ha dado, \\ y la del que su fuego y su locura \\ llora entre aquellas plantas conocidas, \\ apenas en el agua resfriado?
}

Notons tout de suite que "ces plantes connues " le sont de Phaéton mais aussi du lecteur, et cette connaissance préalable que le lecteur a des Héliades exempte d'emblée le poète de tout travail amplificatif.

Dans ce sonnet, le je poétique se trouve plongé dans des affres qui troublent sa faculté de jugement et l'empêchent de prendre la mesure du danger qu'il doit affronter. Leur cause déclarée est "ce désir fou, impossible, vain, craintif ». Le danger, qui consiste à "se laisser accroire ce qu'il ne croit pas ", est assimilé dans les tercets à une chute à travers les évocations successives d'Icare et de Phaéton. Même si aucun destinataire n'apparaît explicitement, force est de reconnaître que la voix poétique s'inscrit ici dans la tradition de la querelle amoureuse.

La démarche argumentative de la voix poétique met clairement en évidence la portée didactique des fables mythologiques : s'il ne lui est pas donné de se voir en elle-même telle qu'elle est, et de considérer le danger qui la guette, aucun exemple, fût-il frappant, ne pourra l'aider. Le verbe " aprovechar » répété dans le deuxième quatrain et le premier tercet fait le lien entre le je dédoublé dans le dialogue qu'il mène avec lui-même et la représentation qu'il pourrait trouver de lui dans les exemples d'Icare et de Phaéton. Soulignons la fonction de miroir assignée à la fois à l'introspection et aux exemples mythologiques, fonction ici inopérante du fait de la confusion qui a gagné le je poétique.

Pour en arriver aux Héliades, elles sont évoquées dans le deuxième tercet, en lien avec le destin tragique de Phaéton, mais dans un raccourci saisissant, tous les éléments étant convoqués en même temps et figés en une seule vision : l'infidélité de Garcilaso au motif ovidien est patente car il programme la chute dans l'Eridan d'un Phaéton pleurant sa destinée au milieu de ses sœurs déjà faites plantes. Or dans le récit d'Ovide, Phaéton, frappé par le foudre de Jupiter, ne survit pas et c'est un corps sans vie que recueillent les Naïades pour l'ensevelir sur le rivage. De même, les Héliades ne se transforment en peupliers qu'une fois découvert le tombeau de leur frère.

Outre l'effet de simultanéité corrigée par une lecture chronologique des éléments constitutifs - effet semblable à celui qui préside aux représentations picturales des récits - la réécriture du récit mythologique par Garcilaso obéit à sa connexion à un topique sous-jacent mais très prégnant qui est celui de la froideur de la dame. Seule la confusion du je poétique avec l'amant - sinon éconduit du moins en proie aux doutes - explique le sort réservé à Phaéton, second avatar de la voix poétique : le désir fou qui les inspire tous deux ne peut les amener qu’à échouer dans leur entreprise, à pleurer leur sort, et à refroidir

2. Garcilaso de la Vega, Poesías castellanas completas, éd. de Elías L. Rivers, Madrid, Castalia, 1996, p. 54. 
leur ardeur. Qu'importe si c'est Phaéton qui se lamente et non les Héliades, qu'importe si Phaéton n'est pas mort au milieu de ses sœurs; qu'importe si les eaux de l'Eridan n'ont pas servi à refroidir le feu qui brûlait son corps, déjà réduit en cendres. Les Héliades sont là à titre d'ornement, importantes en cela qu'elles induisent dans le poème le principe, hautement fructueux dans le discours lyrique, des lamentations.

Mais avant de poursuivre la destinée poétique des Héliades, une incursion vers un autre sonnet de Góngora en lien avec celui de Garcilaso s’impose. À la question rhétorique de Garcilaso, demandant à quoi peuvent lui servir les exemples malheureux d'Icare et de Phaéton, Góngora va en effet composer une réponse poétique, un sonnet exhortant un destinataire tout aussi intrépide qu'Icare à ne pas craindre de prendre son envol, car la renommée vaut bien une fin funeste :

No enfrene tu gallardo pensamiento
del animoso joven mal logrado
el loco fin, de cuyo vuelo osado
fue ilustre tumba el húmido elemento.
Las dulces alas tiende al blando viento,
y sin que el torpe mar del miedo helado
tus plumas moje, toca levantado
la encendida región del ardimiento.
Corona en puntas la dorada esfera
do el pájaro real su vista afina,
y al noble ardor desátese la cera;
que al mar, do tu sepulcro se destina,
gran honra le será, y a su ribera,
que le hurte su nombre tu rüina.

La figure de Phaéton s'est estompée, les Héliades sont absentes, mais reste cette opposition fondatrice dans les vers 6-8 entre la " crainte glacée et la région ardente de la flamme $»^{4}$, image des affres intimes de l'amant pris entre son ambition amoureuse et la peur de l'échec. Demeure aussi la corrélation entre l'imprudence juvénile et l'audace amoureuse.

Depuis que les auteurs se sont massivement emparés à la Renaissance de la figure mythologique de Phaéton, le personnage n'a cessé d'incarner - avec certaines nuances - l'hybris classique : de façon générale, comme le résume Guadalupe Morcillo Expósito, cette figure, concomitamment à celle d'Icare, est susceptible d'être convoquée chaque fois que la voix poétique se considère engagée dans " une entreprise quelque peu dangereuse et audacieuse $»^{5}$. Cette

3. Luis de Góngora, Sonetos completos, éd. de Biruté Ciplijauskaité, Madrid, Castalia, 1992, p. 138.

4. Traduction de François Turner : Góngora. Sonnets, s. 1., Imprimerie Nationale Éditions, 1998, p. 37.

5. Guadalupe Morcillo Expósito, «Faetón. Antes y después de Ovidio », Anuario de Estudios filológicos, 2007, XXX, p. 269-280. 
entreprise, toujours liée au principe moral de l'ambition humaine, pourra être d'ordre intellectuel, sentimental ou politique, et donner forme à un discours philosophique, amoureux ou satirique.

Ana Laura Iglesias, qui s'est intéressée aux représentations iconographiques de l'épisode mythologique de Phaéton, précise que "l'appropriation du mythe de Phaéton au XVII ${ }^{e}$ siècle est prolifique et complexe $"^{6}$ et souligne particulièrement le sens politique et la portée métaphysique qu'a pu revêtir le recours poétique à cette figure.

Trois longs poèmes, à la fin du $\mathrm{XVI}^{e}$ siècle et dans la première moitié du XVII ${ }^{e}$, vont illustrer cet intérêt pour la fable de Phaéton : la Fábula de Phaetonte de Francisco de Aldana en 1589, la Fábula de Faetón du Comte de Villamediana en 1629 et Los Rayos de Faetón de Soto de Rojas en 1639. Il s'agit là d'exercices de réécriture poétique de la fable ovidienne, réalisés dans un langage sublime et audacieux qui semble répondre à la témérité de leur sujet mythologique; c'est notamment ce qui ressort de la lecture de la version de Villamediana à laquelle Góngora a consacré un sonnet élogieux. Selon Ana Laura Iglesias, leur point commun est qu'ils «mettent tous l'accent sur l'échec, la chute et l'ambition humaine, contribuant ainsi à la topique de l'excès ${ }^{7}$. Je ne commenterai pas ces poèmes, qui suivent le développement narratif de la fable dans ses différents épisodes (y compris celui de la métamorphose des Héliades) et recourent aux procédés de l'amplification, pour m'intéresser plus particulièrement aux modalités d'insertion de la figure de Phaéton et des Héliades dans la forme ramassée du sonnet.

L'intégration d'éléments métamorphiques dans la forme du sonnet a ceci d'intéressant qu'elle oblige à une extraordinaire concision allant apparemment à l'encontre de sa nature : rien n'est moins aisé que de saisir la transformation d'un corps en un autre corps, cet " entre-deux où une forme disparaît et où

6. "La apropiación del mito de Faetón durante el siglo XVII es prolifica y compleja. [...] En varios casos-esto se ve con mayor claridad en la literatura-se puede afirmar que la recurrencia a la fábula de Faetón guarda relación con la vida politica de los estados absolutistas, pues en muchas oportunidades Faetón evoca o bien el poder absoluto de la figura monárquica o bien una imagen fracasada que en tanto parodia, constituye una crítica ácida a las pocas capacidades del rey para dirigir y "conducir" el gobierno de la cosa pública. Durante este siglo, el mito se construye a partir de la contraposición de imágenes y ofrece material para distintas metáforas y alegorías. Sus simbolismos se utilizan para abordar problemas metafisicos y religiosos, que se asocian a una preocupación por la condición finita del hombre, por lo fugaz y transitorio de la vida terrenal, aspecto que se visualiza en la ilustración de sarcófagos. La preferencia por este motivo se manifiesta particularmente en la literatura española, donde deben citarse como autoridades las obras de Aldana, Villamediana, Soto de Rojas y Calderón entre otros. Todos ellos ponen énfasis en el fracaso, la caida y la ambición humanas y asi contribuyen a la configuración de la tópica del exceso. " Ana Laura Iglesias, "El mito de Faetón: sobre el exceso, lo fugaz y la conducción. Reflexiones sobre el relato y su apropiación ", Modernidades, revista electrónica de la Universidad Nacional de Córdoba (Argentina), 2006, n 4.

Page web : http://www.ffyh.unc.edu.ar/archivos/modernidades_a/IV/DEFINITIVOS/ Articulo_Iglesias.htm\#_ftn3

7. Ibid. 
un nouveau corps apparaît ${ }^{8}$ selon les termes de Roberto Calasso. Autant dire que la métamorphose s'inscrit dans une temporalité et qu'à ce titre le procès narratif sera le plus indiqué pour saisir la métamorphose dans son déploiement physique. À l'ampleur événementielle et à l'expansion formelle, le sonnet oppose sa brièveté et sa compacité qui contraignent, pour qui s'y astreint, à l'élection, à l'allusion et à l'élision.

S'agissant de l'héritage partagé et archiconnu des métamorphoses ovidiennes, la référence allusive ne peut que très bien fonctionner pour un public lettré : il suffit d'un indice clairement identifiable pour resituer l'épisode mythologique et reconnaître sa portée symbolique. Plusieurs sonnets aux $\mathrm{XVI}^{\mathrm{e}}$ et $\mathrm{XVII}^{\mathrm{e}}$ siècles mettent en scène l'intrépidité de Phaéton et la chute de son char selon des perspectives fort différentes.

Le premier sonnet est celui de Hernando de Acuña :

Con tal instancia siempre demandaba

el gobierno del sol por solo un día,

que, aunque no convenirle conocía,

Febo al hijo Faetón se lo otorgaba.

Ya el carro y los caballos le entregaba

con que la luz al mundo repartía,

poniéndole delante el mal que habria

si en el camino o en el gobierno erraba.

Mas él, de la oriental casa salido, fue el orbe y hemisferio traspasando con furia y con desorden tan extraña,

que el carro, los caballos y él, perdido, sobre el lombardo Po cayó, abrasando riberas, aguas, montes y campaña?

Les sept imparfaits et le mot día, qui s'étirent obstinément à la rime des quatrains pour dire la longueur et la récurrence des tergiversations entre Apollon et son fils, préparent la brutalité du dénouement. Dans les tercets, en effet, les deux prétérits (fue et cayó) précipitent les phases de la catastrophe (traspasando, abrasando) tandis que l'accord de cayó avec le sujet le plus proche (él) engloutit grammaticalement char et chevaux dans la chute du seul Phaéton.

Francisco de Aldana évoque aussi Phaéton dans un de ses sonnets :

IX -

¿Cuál nunca osó mortal tan alto vuelo subir o quién venció más su destino, mi clara y nueva luz, mi sol divino, que das y aumentas nuevo rayo al cielo,

8. Propos de Roberto Calasso rapportés par Hélène Vial dans La métamorphose dans les "Métamorphoses» dOvide, Paris, Les Belles Lettres, 1995, p. 25.

9. Hernando de Acuña, Varias poesías, éd. de Luis F. Díaz Larios, Madrid, Cátedra, 1982, p. 344. 
cuanto el que pudo en este bajo suelo -joh, estrella amiga!, ;oh, hado peregrino!los ojos contemplar, que, de contino, engendran paz, quietud, guerra y recelo?

Bien lo sé yo, que Amor, viéndome puesto do no sube a mirar con mucha parte olmo, pino, ciprés ni helado monte,

de sus ligeras alas dióme presto dos plumas y me dijo: "Amigo, guarte del mal suceso de Ícaro o Faetonte " ${ }^{10}$.

Il s'agit d'un sonnet amoureux où la destinataire se fait nouvel astre, capable de susciter un certain chaos émotionnel chez qui la contemple. Le je poétique, qui s'est abandonné à cette contemplation, est mis en garde par l'Amour luimême, qui l'enjoint à ne pas suivre l'exemple d'Icare ou Phaéton ; les deux plumes qu'il remet en même temps à la voix poétique doivent le préserver de la chute prévisible. L'on comprend dans ce sonnet ce que le projet poétique gagne à additionner les deux figures mythologiques en actualisant des éléments propres à chacun : le chaos pour Phaéton, et les plumes pour Icare.

Un sonnet de Juan de Tassis, comte de Villamediana, reprend ces deux figures :

- VI - // 272

No desconozco en vos, mi pensamiento, para tanta razón, tanta osadía, mas no siempre Fortuna ha de ser guia de tan precipitado atrevimiento.

Ícaro en vano se fió del viento, Faetón regir en vano el sol quería, ventura, y no razón, vence porfía, sólo ventura no es merecimiento.

No os turbe, pensamiento, en la subida, del lastimoso ejemplo en la memoria, ni en peligro mayor, menos ventura;

pues Fortuna que ayuda a la caida, no os podrá quitar aquella gloria de venir a caer de más altura ${ }^{11}$.

Villamediana a fréquemment eu recours dans nombre de ses sonnets amoureux aux mythes d'Icare et de Phaéton pour fustiger l'audace et l'entêtement

10. Francisco de Aldana, Poesías castellanas completas, éd. de José Lara Garrido, Madrid, Cátedra, coll. «Letras Hispánicas », 1985, p. 146-147.

11. Villamediana (Conde de), Juan de Tassis y Peralta, Poesía impresa completa, éd. de José Francisco Ruiz Casanova, Madrid, Cátedra, 1990, p. 352. 
amoureux, l'ambition folle et démesurée appelant souvent un châtiment ${ }^{12}$. Le sonnet retranscrit ici présente la particularité d'une certaine intellectualisation de la dynamique envol-chute : la voix poétique apostrophe sa "pensée " pour la convaincre que la Fortune ne peut à elle seule être la maîtresse de son destin. Le sonnet se construit sur une dualité fortune / raison selon une argumentation qui défend l'œuvre de la raison dans tout dessein élevé, en rejetant les exemples désastreux d'Icare et de Phaéton au prétexte qu'ils ont été victimes de la seule adversité. Et si la voix poétique ne peut s'affranchir de la loi aveugle de la Fortune, au moins doit-elle avoir la certitude de gagner une plus grande gloire à tomber de plus haut. C'est ainsi que la raison engagée dans une noble entreprise peut infléchir le destin. La conceptualisation du thème mythologique et la réflexion sur la raison revêtent des accents néo-platoniciens ; il est de même difficile de ne pas discerner en transparence derrière les figures d'Icare et de Phaéton la célèbre image du Phèdre présentant l'âme comme un char où la raison, installée à la place du cocher, commande aux deux chevaux attelés que sont volonté et désir.

Dans un sonnet de Soto de Rojas, comparable à celui de Villamediana, la voix poétique apostrophe pareillement sa "superbe pensée ", lui demandant où elle est en train de s'envoler :

\author{
¿Dónde vuelas, soberbio pensamiento? \\ Ícaro mozo, mi consejo espera: \\ mira que al polvo humilde y blanda cera \\ ni el sol perdona, ni respeta el viento. \\ Fénix es sol, y su divino aliento \\ la procelosa de Aquilón esfera; \\ de cera y polvo tú porción ligera; \\ teme, vuelve a la tierra, que es tu asiento. \\ Pero sube, camina, no repares, \\ rompa tu fuerza los contrarios vientos \\ hasta ver de tu sol su luz a solas; \\ que, si muerto cual Ícaro bajares, \\ nombre darás al mar de mis tormentos \\ $y$ eterno vivirás entre sus olas.
}

On retrouve les mêmes procédés que chez Villamediana (le dédoublement interlocutif du je à travers l'adresse à la pensée, l'exemplarité des figures mythologiques et la compensation finale de la gloire, le parallèle explicite avec le destin du je poétique) mais ces procédés habillent ici la seule figure d'Icare ${ }^{13}$.

12. Cf. Carlos Mata Induráin, « Neoplatonismo en la lírica del Siglo de Oro. Dos sonetos del Conde de Villamediana ", Anuario Filosófico, revista digital de la Universidad de Navarra, 2000, $\mathrm{n}^{\circ} 33$, p. 641-653. Page web : http://hdl.handle.net/10171/456

13. Cf. John H. Turner, "Notas. Góngora y un mito clásico ", Nueva Revista de Filología Hispánica, 1974, XXIII, nº 1, p. 88-100. 
Ces quelques exemples montrent à quelle perfection est arrivée la fusion entre les figures de Phaéton et d'Icare, dont les évocations fondamentalement liées à la topique de l'hybris se rapprochent plus d'une allégorie désincarnée que d'une représentation littérale des figures mythologiques. Phaéton et Icare sont devenus des signes redondants aptes à se superposer ou à se substituer l'un à l'autre. En outre, il est plus question dans ces quatre sonnets de l'ambition et de l'audace de la pensée (qu'elle soit pensée amoureuse ou faculté conceptrice et projection) que de celles du désir telles que les exprimait Garcilaso. Dans ces sonnets, bien que très différents dans leur facture et leur projet esthétique, s'expriment avant tout des préoccupations philosophiques.

Avec les larmes des Héliades va pouvoir se faire entendre une tonalité proprement lyrique. Les sœurs éplorées vont en effet faire émerger, dans des sonnets amoureux, la désillusion, le dédain et la plainte.

Dans les deux sonnets suivants, Góngora renoue avec la figure plurielle des sœurs de Phaéton, pour en faire le sujet central de sa composition :

Verdes hermanas del audaz mozuelo por quien orilla el Po dejastes presos en verdes ramas ya y en troncos gruesos el delicado pie, el dorado pelo,

pues entre las rüinas de su vuelo sus cenizas bajar en vez de huesos, y sus errores largamente impresos de ardientes llamas vistes en el cielo,

acabad con mi loco pensamiento, que gobernar tal carro no presuma, antes que le desate por el viento

con rayos de desdén la beldad suma, $y$ las reliquias de su atrevimiento esconda el desengaño en poca espuma.
Gallardas plantas, que con voz doliente al osado Faetón llorastes vivas, y ya sin invidiar palmas ni olivas, muertas podéis ceñir cualquiera frente, asi del Sol estivo al rayo ardiente blanco coro de Náyades lascivas precie más vuestras sombras fugitivas que verde margen de escondida fuente,
$y$ asi bese (a pesar del seco estio) vuestros troncos (ya un tiempo pies humanos) el raudo curso deste undoso río,
que lloréis (pues llorar sólo a vos toca locas empresas, ardimientos vanos) mi ardimiento en amar, mi empresa loca ${ }^{14}$.

Comme souvent chez Góngora, l'objet poétique n'est pas directement désigné par son nom propre conventionnel. Les Héliades sont ici interpellées au moyen de périphrases qui prennent immédiatement sens grâce à l'évocation de Phaéton - lui-même caractérisé de façon intransitive par sa jeunesse et son intrépidité dans le premier sonnet et à ce titre aisément reconnaissable. L'apostrophe aux « vertes sœurs de l'intrépide garçon » ou aux " gracieuses plantes qui pleurèrent d'une voix plaintive l'intrépide Phaéton " est ainsi immédiatement contextualisable dans le panorama de la culture mythologique classique. Dans l'un et l'autre cas, la voix poétique enjoint les Héliades à considérer sa folle entreprise.

Le premier sonnet va développer autour des Héliades une série de liens analogiques à partir de l'image du char assimilé à la pensée : le recours aux Héliades ne semblerait être qu'un prétexte (cantonné au premier quatrain) pour

14. Luis de Góngora, op. cit., p. 130. 
que la voix poétique en vienne finalement par ce biais à traiter dans les tercets une fois de plus la représentation archiconnue de l'hybris dans sa version amoureuse.

Le deuxième sonnet, sans se départir de cette représentation classique, toujours présente, s'en éloigne sensiblement dans son organisation interne : le sonnet se centre réellement sur la figure des Héliades pour repousser l'évocation de l'hybris dans les tout derniers vers. Est mise en avant la déploration, très présente dans le lexique (le seul verbe «llorar » est répété trois fois).

L'introduction de la figure des Héliades présente un intérêt poétique majeur : outre le motif des lamentations déjà commenté, elles permettent de donner une réalité poétique à la métamorphose en tant que processus corporel. La gageure que représente l'évocation de la métamorphose dans un sonnet a à voir effectivement avec l'introduction d'une temporalité qui s'accorde $a$ priori difficilement à cette forme brève.

Dans le premier sonnet, la métamorphose des corps est exprimée dans la dénomination périphrastique des Héliades, qui intègre les notions de changement et de permanence puisqu'il est dit en un syntagme nominal que les sœurs de Phaéton gardent cette qualité même après leur transformation physique. Par ailleurs, dans le même quatrain, la voix poétique a recours à une chronologie explicite exposant un après et un avant, où les éléments transformés sont antéposés : « jadis, en verts rameaux, en troncs épais, / leur pied délicat, leur boucle dorée $\|^{15}$. Dans les mêmes vers, notons la construction en chiasme où s'opposent les notions de grossièreté et de délicatesse et où les verts rameaux sont à mettre en relation analogique avec les cheveux dorés, et les troncs épais avec les pieds délicats. De fait, la métamorphose est ici formellement abordée à la fois dans une successivité qui différencie des états préalable et postérieur, et dans une simultanéité qui recompose une réalité complexe faite de traces, d'invariants et de nouveautés. L'unité au terme des différentes transformations (les Héliades transformées en arbres, Phaéton transformé en cendres) et projections analogiques (la voix poétique se projetant en Phaéton précipité si les Héliades ne le ramènent pas à la raison) est assurée par leur développement au sein d'une phrase unique qui ramasse ainsi les événements en un instantané syntaxique.

Dans le deuxième sonnet, le processus métamorphique introduit par la figure des Héliades se donne à voir différemment, de façon plus littéraire - au sens propre - car le motif végétal lui-même est soumis à variation : les Héliades définitivement transformées en plantes (la voix poétique définit d'emblée un avant où elles sont vivantes et un après où elles sont mortes) sont destinées à devenir une ombre protectrice pour les Naïades et à voir leurs troncs baignés par le fleuve. Il est remarquable qu'apparaissent des éléments communs au précédent sonnet mais dans une totale reconfiguration : si par exemple les troncs sont bien évoqués comme ayant été jadis des pieds humains, les rameaux sont évoqués de façon elliptique dans la concurrence qu'ils peuvent faire désormais à la palme ou à l'olivier. De même le « rayon ardent » n'est plus celui

15. Trad. de F. Turner, op. cit., p. 25. 
du foudre de Jupiter (ni du dédain de la dame) mais celui d'un soleil estival dont il convient de se protéger. Les Héliades métamorphosées participent ainsi de la représentation d'un locus amoenus bien éloigné de la primitive scène de déploration aux tonalités tragiques. Quant aux Naïades, elles sont là non plus pour recueillir les cendres de Phaéton (ainsi le racontait Ovide) mais pour jouir amoureusement de ce nouveau lieu naturel habité par les plantes que furent les Héliades. En résumé, la généralisation qui veut que les Héliades aient vocation à pleurer l'issue malheureuse de toute entreprise audacieuse se trouve dans la droite ligne de l'assimilation de l'acte de Phaéton à un orgueil démesuré ; ce qui est inédit, c'est l'insertion d'un espace, lieu amène, qui vient nourrir le propos sentimental. Le poème met en scène l'ambivalence de la figure plurielle des Héliades qui ont vocation à déplorer les "folles entreprises" amoureuses et à abriter leur éclosion et ce, semble-t-il, dans un cercle sans fin.

Au terme de cet itinéraire poétique en quête des Héliades, je rappellerai que le recours conventionnel à un motif métamorphique, loin de figer son sujet dans une doxa poétique, un discours convenu, oblige à le soumettre à toutes sortes de variations. S'agissant du sonnet et de l'évocation elliptique, Hélène Vial rappelle que " les Métamorphoses sont constellées de ces fragments qui mentionnent en passant, sans la raconter, une métamorphose, ce qui représente une forme de variatio tout aussi importante que l'expansion poétique $"^{16}$. La brevitas à l'œuvre dans l'allusion est à mettre en lien avec une esthétique de la surprise et une poésie illusionniste, pour reprendre les expressions d'Hélène Vial : précisément, l'étonnement du lecteur naitra de la confrontation entre resserrement de l'expression et temporalité contrainte, confrontation qui aboutit à une "volatilisation narrative de [la] métamorphose ».

En outre, le sonnet qui intègre ces éléments ne se place pas uniquement dans une tradition qui l'exempterait de tout effort explicatif, il développe tacitement son propre récit en mettant en avant non plus une chronologie mais un point de vue. Dans le sonnet, l'élément métamorphique n'est pas condamné à disparaître mais bien à devenir plus volatil ; la désignation des Héliades comme "plantes " chez Garcilaso, chez Góngora, les ramène à leur état transformé, qui n’abolit pas le récit tacite de leur transformation. De même la douleur des Héliades aux accents si tragiques chez Ovide est sublimée lorsqu'elle est appelée à servir le propos d'un sonnet amoureux. La douleur de la perte, si intrinsèquement liée à la souffrance des corps en mutation dans le modèle ovidien, se désincarne chez Góngora; et c'est lorsque la métamorphose est le plus expressément dite que le poète prolonge le processus métamorphique en versant définitivement le motif du côté de l'aménité. 
